RELEVANT CONSIDERATIONS

The actual process of establishing a PCM-DPM interconnection involves considerations of hardware, software, and system reliability. With respect to hardware, the particular peripherals required for the PCM and DPM will depend on the particular machines. In general, the simplest and least expensive approach is to make the PCM resemble a remote terminal of the DPM, using a dial-up system which includes an asynchronous serial line interface and modems. For PDP-8 series PCMs, this involves only off-the-shelf equipment, and relatively complete information about what is required can be found in the $D E C$ Communications Equipment Handbook available from Digital Equipment Corp., Maynard, Massachusetts. The existence of requisite hardware at the DPM end is a precondition for any consideration of interconnection because this equipment will generally be too expensive to be supplied by the user of the PCM.

Software to service the interconnection is very straightforward for PDP-8 series PCMs: The interconnection is programmed exactly like a teletypewriter. Information is sent to the DPM character by character and received from the DPM in the same fashion. Input programming should take advantage of the interrupt facility to avoid possible loss of characters due to overwriting by the DPM before the PCM has fetched the previous character.

Software for the DPM will depend on the particular time-sharing system in use. In most cases, information will be transmitted and received simply by using the equivalent of FORTRAN "read" and "write" statements referencing the PCM terminal as the device. It is highly desirable, however, that the overall DPM time-sharing system have certain characteristics. Chief among these is some variant of a "virtual machine" concept (cf. IBM 360 Cambridge Monitor System or DEC PDP-10 software system). In such a system, each time-sharing user appears to have the entire DPM at his disposal and powerful macros are available for using the DPM. Furthermore, the system is designed to protect user files against system "crashes," to respond quickly to action at the terminal, and to minimize connect charges.

Some conditions based on DPM system reliability must be attached to the advisability of choosing to interconnect a laboratory's PCM with a central computing facility's DPM. The DPM installation should be expected to remain stable both in hardware and software for the foreseeable future. The DPM time-sharing system must be available for a sufficient number of hours each day at the appropriate times. Cooperation of systems-wise DPM personnel is necessary both in establishing and using the interconnected system. Under these conditions, a hybrid PCM-DPM system can be a powerful laboratory tool of reasonably low cost.

\title{
AVALA, a small on-line system with off-line communication with a large computer
}

\author{
JOSEPH L. YOUNG \\ State University of New York at Stony Brook, Stony Brook, New York 11790
}

When I first heard about this conference, I knew I wanted to attend it because I use an on-line computer in my own research and was interested in the insights of others regarding this important area of instrumentation and experimental technique. I was less certain that I wanted to give a presentation. After all, I had not designed a computer, I had not discovered a new hardware wrinkle, and I had not made any major software innovations. All I had was an idea, which had been implemented in our on-line system, of how communication between a small on-line system and a large central campus-wide facility could be effected and the conviction that such communication could eliminate many problems commonly griped about by users of small on-line systems. Since I did not know if my idea was novel enough to present, I turned to Professor Tepas, the conference director, for guidance; he felt it was, and so $I$ am here today. Thus, if you get great insights from my talk, thank me, but if you are bored, blame him.

Briefly, the idea is as follows: Include in the on-line system one or more magnetic-tape drives compatible with the central campus facility (at Stony Brook, an IBM 360/67). This meant, for us, 9-track 800 -bpi drives with interrecord gaps within IBM tolerances.

The total system configuration can be described quite simply. It was manufactured to our specification by Infotec, Inc., of Westbury, New York, and we call it AVALA, Automated Verbal Associative Learning Apparatus. The core computer is a PDP-8/L with $4 \mathrm{~K}$ memory and standard Teletype reader-punch. Interfaced to it is a tape-drive controller with two tape drives, completely IBM compatible, but limited to tape reels of 7 in. or smaller; communication between the tape drives and the $8 / \mathrm{L}$ is via the data break and bypasses the accumulator. In another room, connected by cables to the main console, is the subject station. It consists of a stimulus display and a response keyboard. The stimulus display is a unit with two banks of eight Burroughs NIXIE tubes each, one bank atop the other; each NIXIE has 14 individually addressable filaments which can be lighted, so that all alphanumeric characters, many special characters, and all manner of stick figures can be presented. There are two response keyboards that can be used. The first is a Teletype Model 33 keyboard, which is simply a keyboard that does not make a hard copy of what is typed on it but only transmits it. The second is a response panel with four buttons. Our PDP-8/L is connected by cable to another one with $8 \mathrm{~K}$ memory and a high-speed reader-punch, enabling us to use those facilities if necessary.

What does this arrangement buy us? Briefly, it gives us the full power of the IBM $360 / 67$ to create sequences of stimulus materials, which are read off the tape on one of the drives, and to analyze the data, which are collected on a tape on the other drive. These capabilities are important to me. I run verbal learning experiments in which temporal and procedural variables are varied within the same S's trials. The algorithms I use to set the sequence of trials for a simple $S$ are quite complex, using much of the available core storage on the $360 / 67$; moreover, they are not linear in that the content of a later trial may well be determined before that of an earlier trial. Furthermore, the analyses I run are often quite complex; I typically run grid searches for the set of parameters of a model giving the best fit to a set of data, a time-consuming process even on the $360 / 67$. Thus, I must be able to communicate effectively with the large computer or else beef up the core 
storage of the on-line computer beyond economical limits.

Now that you have been convinced that, for my purposes and doubtless for many of yours, communication with the central campus facility is essential, why do it off-line via compatible magnetic tape? Why not do it on-line, as Aiken and Millward have, or via a cheaper off-line medium? At Stony Brook, and doubtless at other institutions, a data link to the central facility is dangerous. It ties you to the vagaries of scheduling, down time, routine maintenance, etc., in the central facility at the best of times, and puts you at the mercy of unsympathetic computer center administrations, financial stringencies, etc., at less good times. Baldly put, a data link can only work at those times when both ends of the link are willing to turn it on. I am not ready to trust any computer center that much, and I quite prefer the role of standard batch-processing user. Cheaper off-line media are unfeasible in general. Cards bend, tear, warp, jam machines, get out of order, and go through keypunch machines incredibly slowly. Punched paper tape is refused handling in many computer centers and despised for its slow-down characteristics by the rest. Hand transcription is slow, unreliable, and costly.

So, if you need large core memory capacity for experimental setup or data analysis, but only a small capacity to actually run the experiments, I urge your consideration of a magnetic tape-drive system compatible with your central campus computer.

\section{Hardware and software considerations in using}

\section{a LINC-8 computer in a behavioral toxicology laboratory*}

\author{
G. A. VAN GELDER and RON MUNSINGER \\ College of Veterinary Medicine, Iowa State University, Ames, Iowa 50010
}

A LINC-8 ${ }^{1}$ computer is utilized in the Behavioral Toxicology Laboratory for data acquisition and analysis from electrophysical and behavioral experiments and for real-time control of behavioral experiments. Two examples are given below.

In one experiment, the computer was used to present a conditioned stimulus (CS, tone) to a sheep upon detection of simultaneous high-amplitude, slow-wave spindles in two leads of cortical electroencephalogram (EEG). The sheep was placed in the operant chamber, and four leads of EEG were radiotelemetered to the control room. A paper record of the EEG was obtained on a polygraph. The four EEG signal leads were connected to the inputs of the computer analog-to-digital converter (A/D). The tone source was coupled to one of the computer relays. The frequency and amplitude EEG spindle criteria were set at the console and could be varied while the program was running. Two of the four EEG leads were selected at the console for on-line, slow-wave,

*Development supported by NIH Grant RO1-ES-00122 and EPA Community Pesticide Studies Program Contract 63-03-0036. dual-processor machine with two accumulators, memory buffers, memory address registers, instruction registers, and program counters. The core memory is shared between two processors. The one processor can function as a stand-alone PDP-8. Interfacing to external devices is done through the PDP-8 processor, and the interface described below is applicable to any PDP-8 computer. The advantage of using the LINC processor is in a more advanced instruction set and direct access to the display oscilloscope, relays, sense lines, and LINC tape.

The LINC-8 used in our experiments is equipped with a $32 \mathrm{~K}$ disk, incremental tape, and user-designed interface. ${ }^{2}$ The incremental tape is used to transfer large blocks of data (10-50K) to a larger machine for data analysis. The disk is used for storing programs, subroutines, and data during a run. The interface consists of two independent 12-bit buffered clocks. Each clock is driven by a multivibrator with one of four clock rates selected by a front panel control. Each clock can generate a program-interrupt request and can be preset to any value, which facilitates programming countdown procedures. The clocks can also be run with the program-interrupt capability disabled. The behavioral interface is buffered with flip-flops so that external events are stored until the computer is ready to accept the information. This is of importance when two or more independent paradigms are being run simultaneously. When any one of the flip-flops is set, i.e., an external event has occurred, a program-interrupt request is generated and a flag is set. Control of the computer is automatically shifted to the PDP-8 processor, and the interface servicing program entered.

The user determines interrupt priorities in the sequence of instructions which check the device flags. Because of the dual-processor shared-memory structure of the LINC-8, the interface-servicing subroutine transfers the status of the device flags, clock values, and data buffer to specific core locations addressable by the LINC processor. The LINC program determines what external event has occured and what action needs to be implemented. The LINC instruction group, EXC NN, Operation Codes 0740-0777, has been utilized to provide control of the clocks and interface from the LINC processor. Subroutines reside in memory which interpret the particular EXC instruction, generate the necessary PDP-8 IOT instructions, and return control to the LINC processor. For example, EXC O will disable the 